\title{
PAISAJES EN MOVIMIENTO: SENTIDOS DE LUGAR Y PRÁCTICAS INTERCULTURALES EN CIUDADES DE LA REGIÓN DE LA ARAUCANÍA, CHILE
}

\author{
CHANGING LANDSCAPES: SENSE OF PLACE AND INTERCULTURAL \\ PRAXIS IN CITIES OF LA ARAUCANÍA REGION, CHILE
}

\author{
Gonzalo Salazar1,2,3,4, Martín Fonck ${ }^{3,4}$ y Felipe Irarrázaval 2,4
}

\begin{abstract}
La Región de La Araucanía, en términos de paisaje, ha sido históricamente entendida a partir de sus recursos e hitos naturales, principalmente en función de la exaltación de la región como destino turístico y productivo. No obstante, esta perspectiva genera al menos dos problemáticas para comprender las ciudades de la región. En primer lugar, no se hace cargo de las prácticas cotidianas de los habitantes de sus ciudades; y en segundo lugar, tiende a posicionar jerárquicamente una visión de paisaje por sobre otras, invisibilizando las prácticas en el espacio urbano-territorial de la etnia mapuche. Ante esa problemática, se propone una perspectiva no representacional del paisaje, la cual se basa en las prácticas socioespaciales cotidianas, y los sentidos de lugar con que se va construyendo el paisaje de las ciudades de La Araucanía. Metodológicamente se trabaja en las ciudades de Temuco, Villarrica y Angol, en base a caminatas guiadas por los entrevistados, las cuales dan cuenta de cómo los actores entienden y viven el día a día de las ciudades. Se concluye que más allá de una representación estática de paisaje, en ciudades de La Araucanía se dan interacciones interculturales y urbano/rurales particulares, las cuales deben ser consideradas como parte constitutiva del paisaje relacional del cual son parte.
\end{abstract}

Palabras claves: paisaje, teoría no representacional, interculturalidad, ciudades de la Región de La Araucanía.

Due the efforts of Chilean state to promote Araucanía Region as a touristic place, its landscape has been usually represented with nature and immaculate places. However, such representation involves two constraints to understand the region's cities. First, it does not recognize the daily practices of their inhabitants; and second, it locates hierarchically one landscape perspective over others possible perspectives, like the intercultural or Mapuche perspective, among many. Against this background, this work proposes a non-representational insight to the Araucanía's landscape. Non-representational approach highlights daily socio-spatial practices and the senses of place that underpins the landscape of Araucanía cities. The methodology is based on walking interviews around Temuco, Villarrica and Angol. It allows us to understand how actors live and perceive their cities day a day. We conclude that beyond classic landscape perspectives, Araucanía's cities have complex intercultural interactions and strong urban/rural linkages that must be considered as constitutive elements of landscape.

Key words: Landscape, non-representational theory, intercultural studies, cities of Araucanía Region.

A partir de la ocupación de La Araucanía por parte del Estado chileno a finales del siglo XIX, y el consecutivo proceso de ocupación territorial sobre el territorio Mapuche, se comienza a construir un imaginario paisajístico sobre La Araucanía. La instalación del tren y la creación de caminos -entre otros- facilitaron la llegada de empresas productivas y de urbanización creciente (Flores 2012; Pinto 2003), generando nuevas relaciones en el paisaje y una visión representacional y estática sobre este. De tal modo, se desarrolló una visión del paisaje natural como espacio selvático a ser dominado mediante la habilitación productiva del espacio, propio de la sociedad colona (Camus y Solari 2008). Sumada a esta perspectiva, durante el siglo XX se generó una representación estética asociada al turismo. Esta ocurrió por medio de la construcción del imaginario de belleza nacional del paisaje del sur de Chile, incentivado por el Estado desde la Empresa de Ferrocarriles del Estado y en revistas como En Viaje y La Guía del Veraneante (Booth 2010) ${ }^{1}$.

1 Campus Villarrica e Instituto de Estudios Urbanos y Territoriales, Pontificia Universidad Católica de Chile. O’Higgins 501, Villarrica, Chile.gonzalosalazar@uc.cl

2 Centro de Desarrollo Urbano Sustentable (CEDEUS), Los Navegantes 1963, Santiago, Chile. firarrazava@uc.cl

3 Centro de Estudios Interculturales e Indígenas (CIIR), O’Higgins 501, Villarrica, Chile. mfonck1@uc.cl

4 Centro UC de Desarrollo Local, O’Higgins 501, Villarrica, Chile. 
No obstante, estas representaciones respecto al paisaje de La Araucanía presentan límites, al desplegar una visión reducida de la experiencia de las ciudades, al menos por dos motivos. Primero, no se hacen cargo de las dinámicas presentes en la experiencia cotidiana y las prácticas sociales desde donde se ha construido el paisaje; y segundo, jerarquizan y objetivizan una determinada visión de paisaje por sobre otras. Esto ha repercutido fuertemente en la en la construcción del espacio en las ciudades de La Araucanía, en tanto estas representaciones han operado como agentes de invisibilización de las prácticas de la etnia mapuche. No obstante, las dinámicas socioespaciales de los asentamientos urbanos de la región poseen una densa interacción intercultural definida por los flujos de población rural de origen mapuche, así como también de población rural no mapuche.

Ante esta problemática, el presente artículo se propone como objetivo ir más allá de las visiones representacionales del paisaje mediante la exploración del sentido de lugar, el cual permite dar cuenta de la diversidad de prácticas y perspectivas que coexisten y dan forma a paisajes dinámicamente. Se propone una aproximación no representacional al paisaje (Di Giminani y Fonck 2015; Ingold 2000; Massey 2005; Thrift 2008) de las ciudades de La Araucanía, en donde este es entendido en forma dinámica y relacional. Se exploran tanto las valoraciones medioambientales de los contextos urbanos, así como también los procesos de asignación de significado y los sentidos de lugar emergentes de la experiencia de vida, movilidad y uso en el territorio. Esto permite profundizar en su dimensión vivida, imbuido en los diferentes sentidos de lugar, más que sobrerepresentaciones que no necesariamente dan cuenta de las dinámicas socioespaciales que acontecen en las ciudades. En detalle se trabaja en torno a los paisajes en ciudades de la región de $\mathrm{La}$ Araucanía, específicamente en Temuco, Villarrica y Angol. Metodológicamente se trabaja con caminatas guiadas por los entrevistados en las distintas ciudades, para de ese modo comprender cómo el espacio es configurado desde la experiencia de los actores.

La relevancia del trabajo está dada por dos motivos. En primer lugar, la importancia de esta clarificación conceptual sobre paisaje pasa por contribuir a un mejor entendimiento de la complejidad urbana-territorial de las ciudades de La Araucanía y su condición inherentemente intercultural. Las visiones de paisaje en movimiento nos permiten estudiar de forma dinámica las relaciones urbanorurales que se dan en las prácticas interculturales y que finalmente las anula como dicotomías definidas a priori. A su vez, el estudio de las relaciones interculturales que suceden en las prácticas y perspectivas de movilidad urbano-rural en ciudades de La Araucanía ilustran de forma clara cómo el paisaje es una dinámica en constante construcción por medio del proceso mismo de ser habitado. En segundo lugar, las dinámicas interculturales en las ciudades de La Araucanía han sido escasamente abordadas (Aravena et al. 2005). En general, en Chile se ha tematizado sobre la población indígena en las ciudades, pero sobre todo con un énfasis en las áreas metropolitanas (Abarca 2002; Antileo 2007, 2013; Aravena 2005; Thiers 2014). Por lo tanto, se espera contribuir a la comprensión de las formas de relación e interacción que se dan en las ciudades de esta región, las que, debido a su cercanía a la matriz territorial-cultural del pueblo mapuche, tienen complejidades y dinámicas necesarias de ser abordadas desde su particularidad.

El presente artículo se estructura en los siguientes apartados. El primero aborda la discusión de paisaje. Se examinan los aportes de una aproximación basada en los sentidos de lugar que trasciende a conceptualizaciones representacionales de paisaje. El segundo apartado describe las herramientas utilizadas en el estudio, señalando las contribuciones de una aproximación al paisaje desde entrevistas caminando por las ciudades de estudio. El tercer apartado desarrolla una caracterización general sobre las relaciones interculturales urbanas y da cuenta de dinámicas que son particulares de las ciudades de estudio (a diferencia de dinámicas interculturales en la metrópolis santiaguina). El cuarto apartado desarrolla una discusión más detallada basada en la descripción y análisis de prácticas socioespaciales e interculturales que definen paisajes no-representacionalmente en cada uno de los casos.

\section{Hacia un Paisaje desde los Sentidos de Lugar y la Territorialización de las Prácticas Interculturales}

El paisaje ha sido predominantemente entendido como una representación de la naturaleza objetiva y externa a la mirada que le da forma. En un contexto clásico, ha sido vinculado a una representación artística, fruto de la perspectiva generada desde la mirada humana, y especialmente vinculada a 
lo pictórico. El desarrollo de la perspectiva se sitúa históricamente en el renacimiento italiano y se asocia a la emergencia de nuevas relaciones de producción bajo el contexto del capitalismo incipiente, desencadenando en una visión objetivada de la naturaleza (Berque 2009; Panofsky 1991). Estudios sobre el paisaje han complementado esta postura con la representación ideológica de ciertos valores y su reproducción (Cosgrove 1984). Tal situación se puede apreciar en el caso Chileno. Los paisajes de la zona sur del país, particularmente de la región de La Araucanía, se han promovido por parte del Estado de forma representacional y singular, enfatizando su belleza natural ante todo (Booth 2010). Esto ha generado una noción de territorio jerarquizada, invisibilizando en este caso particular las nociones y prácticas indígenas en este.

Una posible analogía a la supremacía de una perspectiva determinada en materia de paisaje se puede encontrar en los debates epistemológicos de la crisis ambiental (Bustos et al. 2015; Leff 2010) y del pensamiento decolonialista (De Souza Santos 2010; Mignolo 2010; Walsh 2012). Con matices diferentes, en ambos debates se caracteriza y critican la suposición de una sola forma válida de construir conocimiento que se impone por sobre otras, basada en una epistemología universalista y objetivista. Frente a esta problemática, Leff (2010:99) propone un descentramiento epistemológico por medio de la "deconstrucción del pensamiento metafísico, científico y postmoderno; de la territorialización de la diversidad, la diferencia y la otredad; sobre la base de los potenciales ecológicos y de los saberes culturales que habitan los territorios del sur". De este modo, la territorialización de las prácticas interculturales ha emergido como enfoque clave para el descentramiento epistemológico (Martínez 2012; Molina 1995). Más allá de un foco centrado solamente en las representaciones discursivas, se trata de comprender el territorio a partir de las prácticas, dando cuenta de las formas en que se produce el espacio.

La territorialización de las prácticas es fundamental para el estudio de la interculturalidad. La clave se encuentra en evidenciar las estructuras de poder y la lógica colonial que subyacen a los procesos de invisibilización de las prácticas y los significados interculturales. El espacio no puede ser comprendido al margen de las prácticas sociales. Los procesos de asignación de significado y los sentidos de lugar son emergentes de las prácticas de vida, movilidad y significado del territorio. Esto, a su vez, hace referencia a la literatura que aborda la interculturalidad desde las reivindicaciones por lo local, la memoria y el territorio desde "identidades basadas en lugar" (Escobar 2008; Maldonado y Del Valle 2016). La noción de territorialización tiene como potencial desde este marco, ensalzar la identidad étnica y la autoidentificación por medio del espacio (Bello 2011). Por lo tanto, la interculturalidad debe ser abordada desde un descentramiento epistemológico que emerge al territorializar la diferencia (Maldonado y Del Valle 2016; Martínez 2012; Salas Astrain 2014).

El énfasis en territorializar la diversidad es elemental para el debate de paisaje, en tanto el espacio como categoría analítica posee una alta potencialidad para identificar diversas formas de comprensión y coexistencia del entorno, razón por lo cual es en buena medida valorado por los estudios ambientales (Aliste y Nuñez 2015; Escobar 2008). Es en ese sentido que las prácticas espaciales son útiles para develar las representaciones del paisaje, así como también para avanzar hacia comprensiones de éste ancladas a la diversidad.

Parte importante de la valoración espacial proviene de los autores asociados al "giro espacial" (spatial turn) (Massey 2005; Soja 1996; Thrift 2008), quienes apuntan a una epistemología anclada a prácticas espaciales y modos de vida contextualizados. De tal manera, las prácticas en el espacio dan cuenta de múltiples interacciones entre diferentes concepciones de espacio, lo que se complejiza aún más cuando suceden en un contexto intercultural. En este sentido, aparecen diferentes capas y dinámicas que generan un paisaje en movimiento. Desde esta perspectiva, se vuelve problemático cuando el paisaje es representado por una sola visión del habitar que invisibilice a quienes cohabitan el mismo espacio. A su vez, es necesario trascender una visión representacional del paisaje. $\mathrm{Si}$ bien existen importantes aportes respecto a los elementos simbólicos del paisaje (Geertz 2000; Sauer 1925), es necesario generar una comprensión en profundidad de las experiencias, prácticas e involucraciones contextuales en la producción del espacio. Es en este sentido que Skewes et al. (2014) sugieren dejar atrás la esencialización representacional del paisaje, pasando de una mirada escénica a una protagónica; de la experiencia visual a la sensorial de sus protagonistas.

Específicamente en la discusión de paisaje, son las acepciones de lugar y de sentido de lugar las 
que permiten darle un fundamento epistemológico acorde al "giro espacial" propuesto. Estas nociones han sido mayormente empleadas desde la geografía y psicología ambiental (Altman y Lowy 1992; Jackson 1984; Manzo y Perkins 2006; Nogué 2008; Proshansky et al. 1983; Tuan 1974), y más recientemente desde la antropología (Di Giminiani y Fonck 2015; Hirsch y O'Hanlon 1995; Ingold 2000; Tilley 1994). "El sentido de lugar" (Feld y Basso 1996; Hirsch 1995; Nogué 2014; Relph 1976; Tuan 1974) es parte de una tradición de investigación desde la cual se pretende atender la experiencia del espacio por medio de los procesos del habitar, explorando en profundidad los sentidos configurados en éste. Esta noción se inspira en la perspectiva del habitar (dwelling perspective) desarrollada por el filósofo Heidegger y asociada en el campo antropológico al trabajo de Ingold (2000). De este modo, el sentido de lugar se vincula al paisaje en la dimensión de lugar vivido, la cual deriva de vertientes "fenomenológicas" de la geografía humana (Relph 1976; Tuan 1974), donde se señala la relevancia que juega la experiencia en la asignación de significados al lugar, en oposición a la abstracción del espacio geométrico. En ese plano, según Low (1992) el sentido de apego al lugar es la relación simbólica dada culturalmente por los significados emocionales/afectivos hacia un espacio particular o un pedazo de tierra que provee la base para el entendimiento individual y grupal de la relación con el medio propio (Low 1992:165).

El sentido de lugar que suscita el paisaje dialoga con su capacidad de agencia, dejando de ser el paisaje una representación fija de adscripción territorial, sino más bien estados que se van articulando en diálogo con los sentidos que se hacen presentes en el paisaje. No obstante, tal como señala Gupta y Ferguson (1992) el sentido de lugar presenta el riesgo de naturalizar la relación entre identidad y lugar, omitiendo las tensiones constitutivas del mismo proceso. En ese sentido, la teoría no representacional (Thrift 2008) es un aporte para la comprensión del paisaje (Corsin 2003; Cresswell 2004; Di Giminani y Fonck 2015) en tanto incita a pensar el espacio de forma asociada a "presentaciones" o instantes donde la vida social se va haciendo presente (Corsin 2003). Más que buscar los significados subyacente al paisaje (como lo sugieren las aproximaciones tradicionales al sentido de lugar), interesa indagar cómo son sociopolíticamente tematizados, expresando tensiones que aparecen en la ciudad desde su dimensión de lugar, reuniendo cosas, pensamiento y memorias en configuraciones particulares (Escobar 2001). En ningún caso los aportes de la teoría no representacional, ni las críticas de Gupta y Ferguson (2008), plantean dejar de lado el sentido de lugar, sino instan a llevarlo más allá de visiones estáticas, enfatizando la continua apertura constitutiva implícita en la construcción del lugar. Tal como lo plantea Cresswell (2004), los lugares nunca están completos, terminados o delimitados sino siempre están comenzando en un proceso:

\section{El lugar es la materia prima para la producción creativa de identidad antes que una etiqueta a priori. Los lugares proveen la condición de posibilidad para la creación de prácticas sociales. El lugar en este sentido se vuelve un evento antes que una seguridad ontológica enraizada en nociones de autenticidad. El lugar es un evento marcado por aperturas y cambios antes de ser delimitado y permanente (Cresswell 2004:39).}

De tal modo, finalmente un foco en el paisaje desde el sentido de lugar nos da pie a profundizar respecto a la relación constante entre la ciudad y su medio circundante, problematizando los conocimientos involucrados en la experiencia directa del paisaje, en tanto se trata de epistemologías y prácticas interculturales ancladas a la vida junto al entorno próximo.

\section{Caminando el Paisaje: Aproximación Metodológica}

Para realizar una aproximación al paisaje y los sentidos de lugar, el presente trabajo se desarrolló tomando como herramienta metodológica caminar entrevistando por la ciudad. Siguiendo contribuciones recientes (Anderson 2004; Evans y Jones 2011; Ingold y Lee 2007) se incentivó explorar los sentidos de lugar y prácticas en el paisaje, abriéndose a las formas de conocer y pensar de las personas entrevistadas por medio de desplazamientos que permiten entrar en una relación de copresencia con el lugar habitado. En el proceso de análisis se busca un involucramiento con los actores entrevistados, por medio de una experiencia in situ y en un contexto geográfico particular. De tal modo, se parte de la base que no se "camina 
hacia" el mundo de otras personas, sino que se "camina con" el otro, compartiendo experiencias y observaciones del entorno local (Ingold y Lee 2007). De esta manera, caminar junto al otro es una práctica de entendimiento de los detalles del lugar y el entorno circundante, siendo el recorrido y la experiencia de este, tan válidos como el discurso que lo acompaña. Así, salir a caminar permite una situación de encuentro con el paisaje y vinculación directa con las prácticas espaciales. Es por medio de estos encuentros con el paisaje que se seleccionan prácticas y lugares que el entrevistado considera digno de atención. Cabe señalar, de todas formas, que el acceso a la experiencia y los discursos se encuentra delimitada por el contexto y la relación que se establece con los entrevistados al llevar a cabo los recorridos. Sin embargo, al realizar los recorridos con las diversas personas entrevistadas, es posible distinguir prácticas y sentidos de lugar inherentemente heterogéneos, como también aquellos que son invisibilizados. En este sentido, es clave para la metodología las diferencias entre los perfiles de las personas entrevistadas.

Respecto de los casos de estudio, las tres ciudades seleccionadas (Villarrica, Angol y Temuco) son los principales asentamientos urbanos en términos demográficos de la Región de La Araucanía y los que configuran los territorios funcionales urbanorurales más complejos. El abordaje metodológico se ha desarrollado durante un período de tres años (2013-2015) mediante una aproximación cualitativa. Esta se ha llevado a cabo con entrevistas caminando por la ciudad, complementadas con 56 entrevistas semiestructuradas. Tal desarrollo metodológico ha permitido, por medio del levantamiento de información junto a las personas identificadas, identificar lugares claves para profundizar respecto de las interacciones espaciales en la ciudad. De tal modo, se ha logrado obtener una mayor profundidad sobre los discursos, prácticas y expresiones espaciales del paisaje en las ciudades de estudio. Los entrevistados pertenecen a organizaciones urbanas locales de las ciudades, tanto públicos como de la sociedad civil e indígenas como no indígenas.

\section{Prácticas Interculturales en el Paisaje: Una Aproximación a la Relación Urbana- Territorial de La Araucanía}

La Araucanía es la segunda región con mayor población rural en Chile, con 32,3\%, y la que cuenta con mayor proporción de población indígena del país, con 32\% (Ministerio de Desarrollo Social 2013). En el presente trabajo planteamos dos alcances asociados a las mencionadas características: en primer lugar, más allá de la alta población rural en la región, se debe entender que estas cifras tienden a representar las condiciones de residencia de la población, y por ende no permiten examinar los procesos de movilidad diaria que esta tiene. La población rural de La Araucanía frecuentemente se moviliza hacia las ciudades por motivos de trabajo, estudio o de otra índole, por lo cual la diferenciación urbanorural es solamente nominal, y no da cuenta de las interacciones territoriales que se dan. En segundo lugar, y estrechamente relacionado con lo primero, se debe atender que la marcada presencia mapuche en la región genera una impronta intercultural en las ciudades, la cual tiende a ser reducida a elementos pictóricos y folclóricos de índole representacional. Sin embargo, en este artículo se postula que la interculturalidad se hace presente en la movilidad urbana rural, en el comercio y actividades cotidianas, y que pese a ser políticamente invisibilizadas en la experiencia urbana, son constitutivas de las ciudades de la región.

Actualmente se estima que el $71,8 \%$ de la población mapuche vive en zonas urbanas a escala nacional (Ministerio de Desarrollo Social 2013), la cual tiende a concentrarse en las áreas metropolitanas del país. En ese sentido, bajo el nuevo emblema de "indígena urbano", los estudios empíricos realizados en Chile en esta materia han estado substancialmente enfocados en áreas metropolitanas, principalmente en Santiago (Abarca 2002; Antileo 2007, 2013; Aravena 2005; Thiers 2014). Sin embargo, en la región de La Araucanía también hay una importante presencia de población mapuche urbana, la cual representa el $35,4 \%$ de la población urbana. Debido a esta tendencia, junto a la marcada impronta mapuche de $\mathrm{La}$ Araucanía, emergen dinámicas interculturales que son inevitablemente constitutivas del paisaje de los asentamientos. No obstante, no se ha profundizado mayormente en las dinámicas interculturales asociadas a las ciudades emplazadas en el territorio ancestral mapuche (Aravena et al. 2005). Esto se vuelve determinante, en la medida que los términos políticos asociados a la demanda mapuche, como autodeterminación, reconocimiento y derechos territoriales (Antileo 2010), dan cuenta de un carácter profundamente arraigado al territorio. 
A diferencia de las ciudades de La Araucanía, en Santiago la noción de territorio se complejiza al encontrarse distanciados del territorio reivindicado, generando diversas postura al interior del movimiento frente a la migración urbana, la identidad territorial y las perspectivas políticas futuras. Tal como señala Bello (2002:43), uno de los mayores problemas a discutir en el contexto de migración, o de los mapuches urbanos, es el que deriva del debate sobre la identidad en contexto territoriales distintos a los originales o históricos. En ese plano, los procesos de etnificación territorial que se dan en Santiago construyen paisajes diferentes a los derivados de los asentamientos de La Araucanía. Como se argumenta en este artículo, la matriz territorial en que se emplazan estos últimos conlleva dinámicas socioespaciales interculturales que generan distinguibles sentidos de lugar y una relación urbana-territorial indivisible.

En el caso de las ciudades de La Araucanía, estas se caracterizan inherentemente por una dinámica intercultural. Aquí la población mapuche urbana no solo se construye por fenómenos de migración-que son ciertamente altos- sino también se hacen parte de complejos procesos de movilidad por trabajo, salud, educación y ocio que se ejercen a diario. Esto genera un contexto particular en el estudio de interculturalidad urbana en esta región, y que se materializa de un modo distinto en el paisaje, en tanto las prácticas interculturales en el espacio urbano se asocian a actividades de desplazamiento que ponen en entredicho representaciones del espacio desde donde se ha entendido lo étnico.

Estas prácticas de las ciudades de La Araucanía tienen dos aristas. La primera es que la proximidad a los territorios ancestrales ha generado que la población mapuche urbana nunca hayan perdido una cercanía cotidiana con los sectores rurales y por ende identitaria, por medio de redes y lazos familiares, de tal modo que "el acceso a la identidad de origen está siempre presente, al alcance de la mano, a una o dos horas de viaje en bus" (Aravena et al. 2005:129). En segundo lugar, tal entramado geográfico es aún más evidente y complejo si se toman en cuenta los procesos de conexión y movilidad entre espacios urbanos y rurales que se efectúan a diario por efectos derivados de conmutación. Esta dimensión es la que más acentúa las dinámicas de interculturalidad, en tanto son fundamentalmente vínculos cotidianos y actividades funcionales entre población de zonas rurales y mapuche, configurando el paisaje en los asentamientos de La Araucanía.

De tal modo, cobran sentido afirmaciones de entrevistados por Aravena et al. (2005) como: "el rostro mapuche de la capital de La Araucanía, sostienen, se asocia en la actualidad a los campesinos que transitan por el sector de la feria Pinto, el terminal rural y sus calles aledañas" (Aravena et al. 2005:126), con un énfasis en la conectividad y la ciudad de Temuco como lugar de paso hacia las comunidades. No obstante, la continuidad urbana-territorial por medio de las prácticas de movilidad con una alta diversidad étnica no implicaría necesariamente un reconocimiento político y territorial de esta. Esto se expresa en las ciudades de estudio, las cuales son ejemplo de una invisibilización de la población mapuche, fruto del origen colonial de sus asentamientos. Bajo estos planteamientos, se vuelve necesario someter a crítica dichos postulados sobre prácticas y visibilización política en ciudades de La Araucanía, realizando una aproximación a la experiencia urbana actual.

Si bien la relación entre lugar e identidad en la cultura mapuche ha sido problematizada en los procesos de migración mapuche -asociados principalmente a la ciudad de Santiago-, el sentido de lugar construido desde el paisaje sigue siendo políticamente central. Es en este contexto que la noción de paisaje en estas ciudades de estudio toma características particulares. No se trata de una representación del territorio desanclada de las prácticas de vida en él. La construcción de la idea de paisaje es cotidianamente constituida por medio del emplazamiento y conexión de la ciudad con su entorno. Esto es importante al pensar la identidad étnica asociada a la vida en el espacio urbano y los desplazamientos hacia las comunidades, como formas particulares de vivir el territorio.

\section{El Paisaje en Ciudades de La Araucanía: Visiones Representacionales v/s Prácticas en el Espacio}

A primera vista, es común encontrarse con las representaciones del paisaje recorriendo las ciudades de estudio junto a los entrevistados. $\mathrm{La}$ vida de los asentamientos urbanos en el paisaje es parte del discurso público oficial y el modo desde donde la ciudad se autoidentifica. En este apartado se puede apreciar una selección y descripción de lo que ha sido entendido bajo el presente argumento 
como "paisaje representacional" en cada una de las ciudades de estudio. Sin embargo, profundizando en los sentidos de lugar, es posible apreciar que el paisaje, antes que una representación separada del espacio urbano, es vivido cotidianamente desde los movimientos que ponen en conexión las ciudades con el territorio circundante. De tal modo, nos acercamos a entender el paisaje como una experiencia de vida que se plasma en lugares concretos: en terminales, estaciones de tren, paraderos y ferias. Esta visión del paisaje nos auxilia en avanzar más allá de visiones estáticas del espacio, las cuales presentan el riesgo de evaluar el paisaje desde su carencia o faltas de conocimiento sin reflexionar sobre las prácticas de habitar el espacio. En este sentido, antes que las características propiamente visuales, que le dan el carácter de paisaje representacional desde la perspectiva de los entrevistados, los casos expuestos señalan principalmente prácticas en el espacio. Se trata de lugares que ponen en conexión y diálogo diferentes territorialidades. De tal manera, la experiencia de vida va aconteciendo, dándole relevancia a las prácticas de movimiento en el espacio, antes que a una lejanía visual, la cual posee el defecto al priorizar una visión sobre otras y no dar cuenta de la vida en el espacio.

\section{Angol: entre la belleza de la orquídea y la transgresión agroforestal}

Al recorrer la ciudad de Angol, para la mayoría de los actores entrevistados la referencia principal es la cordillera de Nahuelbuta, famosa por poseer bosques de araucarias y una biodiversidad única. $\mathrm{Si}$ bien la cordillera es parte del discurso de quienes han sido entrevistados y las instituciones locales de la ciudad, al señalar el entorno natural no es posible ver el parque en el paisaje urbano. En contraste, las autoridades municipales han intentado, por medio de representaciones visuales, integrar la ciudad y el parque. De a poco, se ha intentado asociar la imagen de la ciudad a la cordillera, rescatando la araucaria (especie vinculada a la etnia mapuche) y la orquídea (especie local autóctona) en los emblemas de la ciudad. Se busca que la cordillera sea un ícono que representa a la ciudad, desarrollando estrategias de comunicación mediante folletos y publicidad enfocados hacia el turismo. De tal modo, se fomenta que la condición geográfica al borde de la ciudad provea de sentido urbano, retomando relaciones por medio de representaciones estéticas de la naturaleza.
Sin embargo, la imagen principal de una ciudad vinculada a su "belleza natural" como ícono identitario y turístico, fomenta una representación de paisaje que oculta otras dinámicas y problemáticas socioespaciales presentes en la relación de la ciudad con su territorio circundante. En esta línea, antes que un foco en el carácter representacional del paisaje, puede ser provechoso abordar este mismo a partir de los sentidos de lugar y las prácticas en el espacio.

En la ciudad de Angol existe una invisibilización territorial del componente étnico. Históricamente, en la comuna de esta ciudad, a pesar de encontrarse situada en el territorio mapuche, se tendió a desplazar a la población mapuche hacia otros sectores fruto de su condición de microclima y tierra con vocación productiva, las cuales hicieron del encajonamiento de Angol un sitio ideal para la colonización. Debido a este desplazamiento, ha existido una constante invisibilización en el espacio. Los procesos de reivindicación territorial Mapuche en la zona se han entendido como fenómenos ajenos a la ciudad, como un problema del campo, que sucede dislocado del espacio cívico que tiene Angol como capital provincial. En la práctica, los procesos de reivindicación territorial mapuche se han concentrado más en las comunas rurales anexas a Angol, y en la última década se ha tensionado más con la masificación de la industria forestal en la zona, en lo que hoy se conoce como la "zona roja" del conflicto chileno-mapuche.

Justamente es en el paisaje y los sentidos de lugar relacionados a la industria forestal donde se presenta uno de los principales factores territoriales de la tensión intercultural, los cuales no son ajenos al fenómeno urbano. En la última década, resaltan diversos efectos socioecológicos e interculturales que ha tenido la expansión de la industria forestal en La Araucanía. En los recorridos realizados, mirando desde la ciudad en dirección al Parque Nacional Nahuelbuta no es posible verlo. En cambio, se observan plantaciones de pino (Pinus radiata) y eucalipto (Eucaliptus globulus), especies exóticas al ecosistema local, como lo expresa la Figura 1. $\mathrm{Al}$ encontrarse con este paisaje, las personas entrevistadas plantean que la principal problemática es la falta de agua.

Nosotros lo vemos como la explotación de los terrenos, en el tema forestal, de los cauces de agua que ya poco queda, que 


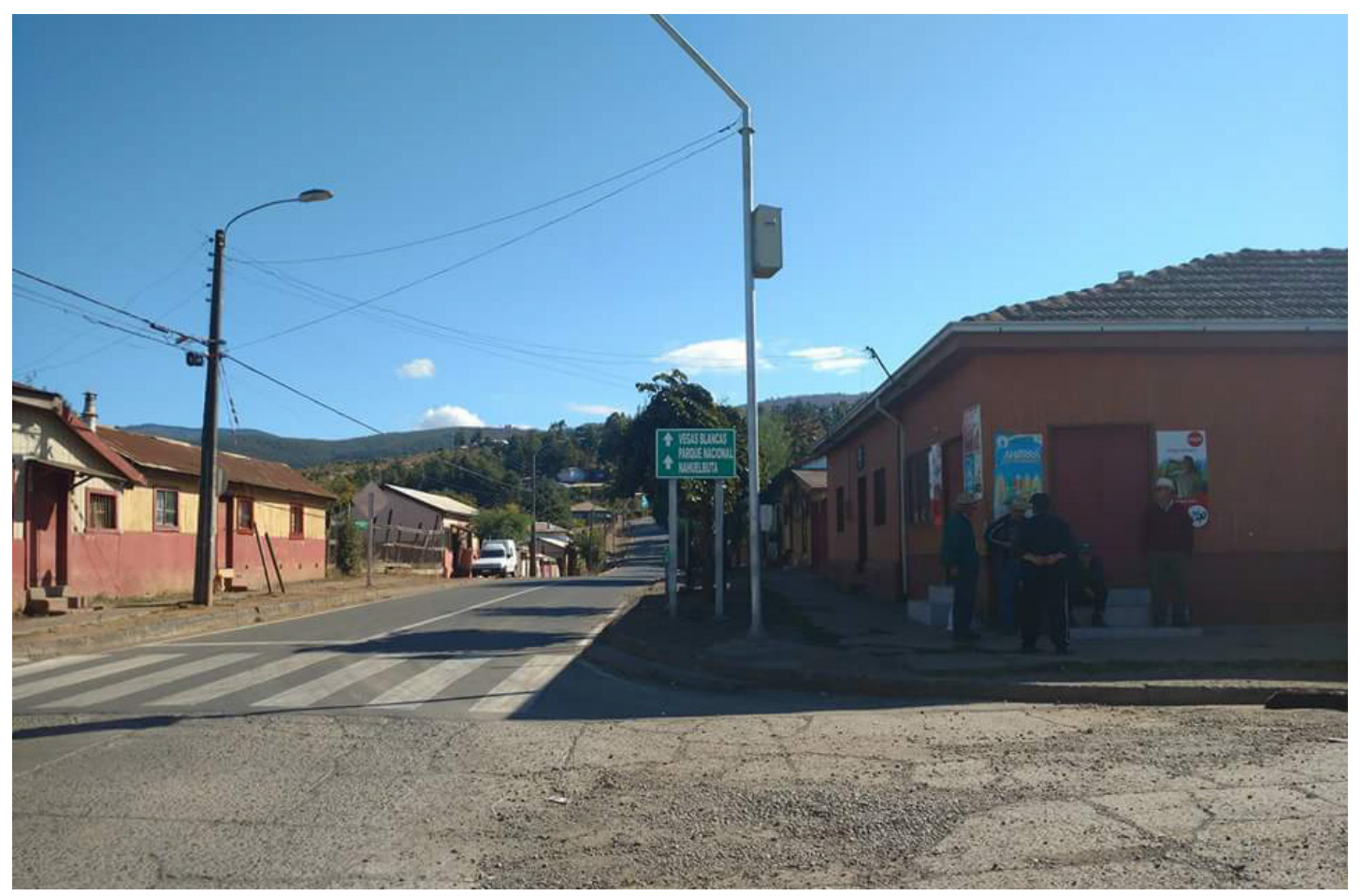

Figura 1. Parque Nahuelbuta desde la ciudad de Angol. Fuente: Álvaro Díaz. Nahuelbuta National Park seen from the city of Angol. Source: Álvaro Díaz.

es uno de los problemas principales que nosotros tenemos en las comunidades, que es la falta de agua por ejemplo, que en el fondo los recursos se han utilizado de tal manera que ya no están disponibles en algunas comunidades, las vertientes que ya no están porque a un metro sembraron eucaliptus (mujer, funcionaria municipal de Angol).

Al recorrer la ciudad y su entorno se da cuenta cómo la experiencia relatada sobre el agua va más allá de una representación del paisaje. La disminución de los esteros observada se plasma en los sentidos del lugar constituidos junto a las transformaciones del paisaje. "¿Y a qué se debe la escasez" (hombre, organización ambiental, Angol). Esta es una pregunta clave que plantea el entrevistado al observar un estero, señalando los usos de las plantaciones en el telón de fondo del recorrido como respuesta. Los sentidos de lugar dan cuenta cómo las transformaciones en los últimos años en el entorno de Angol son transversales al territorio en el cual la ciudad se encuentra emplazada, develando la directa relación entre la demanda mapuche y los efectos territoriales de la expansión forestal.

\section{Temuco: del cerro Nielol a la Feria Pinto, prácticas de desplazamiento interculturales en la ciudad}

El cerro Nielol es un lugar cargado de significados y un paisaje icónico de la ciudad de Temuco. En los recorridos, el cerro es identificado por varios entrevistados como un ícono de la biodiversidad y significación intercultural del paisaje de la ciudad. Recorriendo la cima del cerro se puede apreciar cómo el ambiente físico en el cual se encuentra emplazada la ciudad, ha sido ritualmente dotado de significado por espacios ceremoniales, siendo representado principalmente por los chamemull (estatuas rituales mapuche) en la cima del cerro que aparecen en la Figura 2.

Por medio de las prácticas en estos espacios, se da cuenta cómo el cerro y los elementos de la naturaleza, como ríos y bosques, son espacios centrales para la cosmovisión mapuche. Los aspectos 


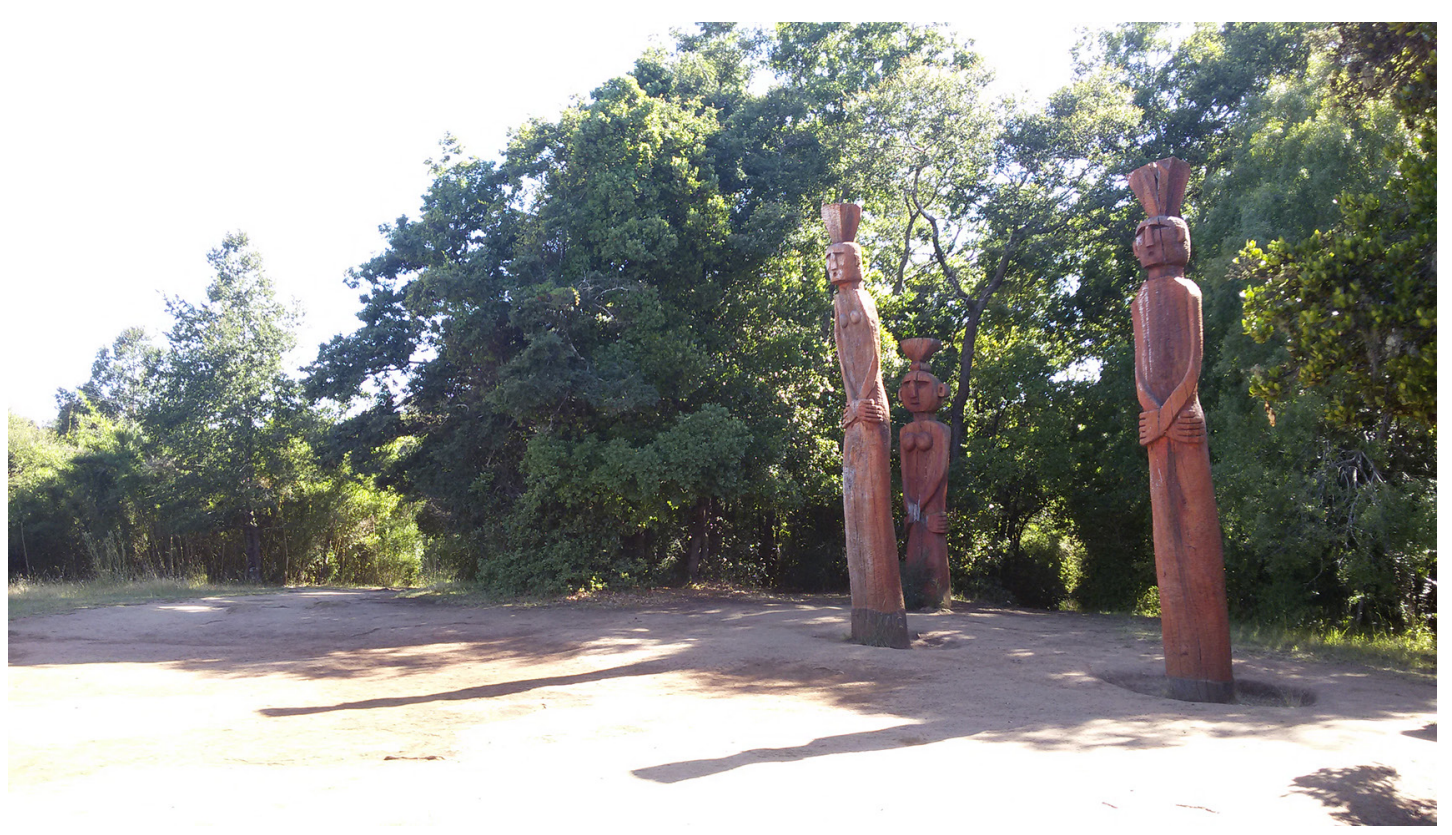

Figura 2. Cima del cerro Ñielol, Temuco. Fuente: Elaboración propia. Summit of Ñielol Hill, Temuco. Source: Own Elaboration.

ceremoniales no son separados del conocimiento y uso del lugar, reflejado en la importancia medicinal que tiene el entorno, por medio del uso de las machis que suben a buscar el lahuen (hierbas medicinales), como se afirma:

Las machis subían al cerro en sus tiempos y hasta el día de hoy lo hacen para buscar lahuén, que son las hierbas medicinales para hacer sus respectivos remedios para sanar enfermedades del cuerpo... en el cerro encontrai diferentes tipos de hierbas, diferentes tipos de vegetación, un espacio ancestral para el pueblo mapuche (hombre, dirigente social, Temuco).

En este sentido, la interculturalidad está dada por las prácticas en el espacio.

Sin embargo, personas pertenecientes a organizaciones de defensa del patrimonio de la ciudad describen que el cerro es invisible para el habitante común de la ciudad, atribuyéndolo a una falta de valoración ambiental desde un diagnóstico propio de una visión de paisaje representacional. En este sentido, si bien el cerro es parte emplazada de la cosmovisión mapuche, reflejando la íntima e indivisible conexión entre práctica ritual y biodiversidad, desde las representaciones predominantes de paisaje es entendido desde su carencia significativa para ciudad en su conjunto. Estos elementos se argumentan al recorrer el cerro, emergiendo juicios respecto al rol visual del cerro en la ciudad y la perspectiva del habitante común: "el cerro es invisible porque es un cerro que pasa desapercibido. Sabemos que está, pero no vamos" (hombre, organización urbana, adulto). Desde este diagnóstico, la complejidad relacional del cerro Ñielol es invisibilizada, siendo evaluado desde una representación estática y patrimonial.

No obstante, es posible pensar el paisaje de la ciudad de Temuco más allá del entorno medioambiental próximo a la urbe, indagando en los espacios de conexión con el mundo rural al interior de la ciudad, tal como la Feria Pinto, donde se viven las prácticas en el espacio. La Feria Pinto es entendida por varias personas entrevistadas recorriendo el espacio como el corazón de la ciudad de Temuco y la región de La Araucanía por el comercio y las prácticas de movilidad relacionadas a esta. Las prácticas y encuentros que emergen desde la feria se caracterizan principalmente por su diversidad étnica, siendo la interculturalidad una característica identitaria del espacio, tal como lo expresa un dirigente social: "la realidad está en el entorno y ahí está la cultura. No 
en lo bonito, en lo que tú haces, en la arquitectura no, en la diversidad está la riqueza de la ciudad... la realidad está aquí y la cultura está aquí en la Feria Pinto porque nosotros tenemos la interculturalidad juntos" (hombre, dirigente social, Temuco). Esto a su vez es acompañado del movimiento presente en productos provenientes de diversos orígenes, marcando las interacciones espaciales en torno al comercio y la presencia de la diversidad biocultural regional. En este sentido, el encuentro está dado por las experiencias de movilidad desde el campo a la ciudad, lo cual se encuentran expresados en los murales de "Alapinta"2 que han retratado esta interacción al interior y exterior de la Feria. En este sentido, es que uno de los dirigentes de la feria señala:

Nosotros estamos aledaños con el terminal de buses rurales po, ahí es donde llega toda la gente... Porque aquí uno mismo ve esta, por ejemplo, si uno mira este cuento, todos estos murales que hay, esto tiene su razón de ser, hay una información en los murales. Cómo se inició la feria, al lado de ferrocarriles, cómo llegaban las mercaderías, las ñañitas que son parte nuestra, el mote, todas esas cosas, pa' allá están los recolectores, a la vuelta, los recolectores de piñones y todo eso. Estos dicen a que a la vueltecita hay un camioncito y una carreta (hombre, feriante, Temuco).

Estas expresiones dan cuenta que el paisaje no es una representación externa a la ciudad, aprehensible desde lo visual y sus reproducciones. El paisaje está dentro de la ciudad, emergiendo constantemente en el desplazamiento, en la interconexión de lugares vividos y en prácticas en el espacio, tal como lo expresa estos murales en la Figura 3.

\section{Villarrica: ¿Palco para mirar el volcán o nodo de movilidad territorial?}

El paisaje, para los entrevistados de Villarrica se presenta en la costanera de la ciudad, acompañando la conversación con una contemplación del contexto natural que permite amplias perspectivas visuales de cerros, volcanes y bosques a lo lejos, tal como lo expresa la Figura 4. Es común comenzar a hablar sobre la ciudad mirando el lago o el volcán, como elementos presentes en la vida cotidiana de la ciudad, viendo el paisaje desde su atractivo visual y potencial turístico. Sin embargo, al comenzar a profundizar respecto de la influencia del hombre en el entorno, en los recorridos con dirigentes vecinales y funcionarios municipales aparece un aire crítico respecto de la involucración de la ciudad con su medio ambiente.

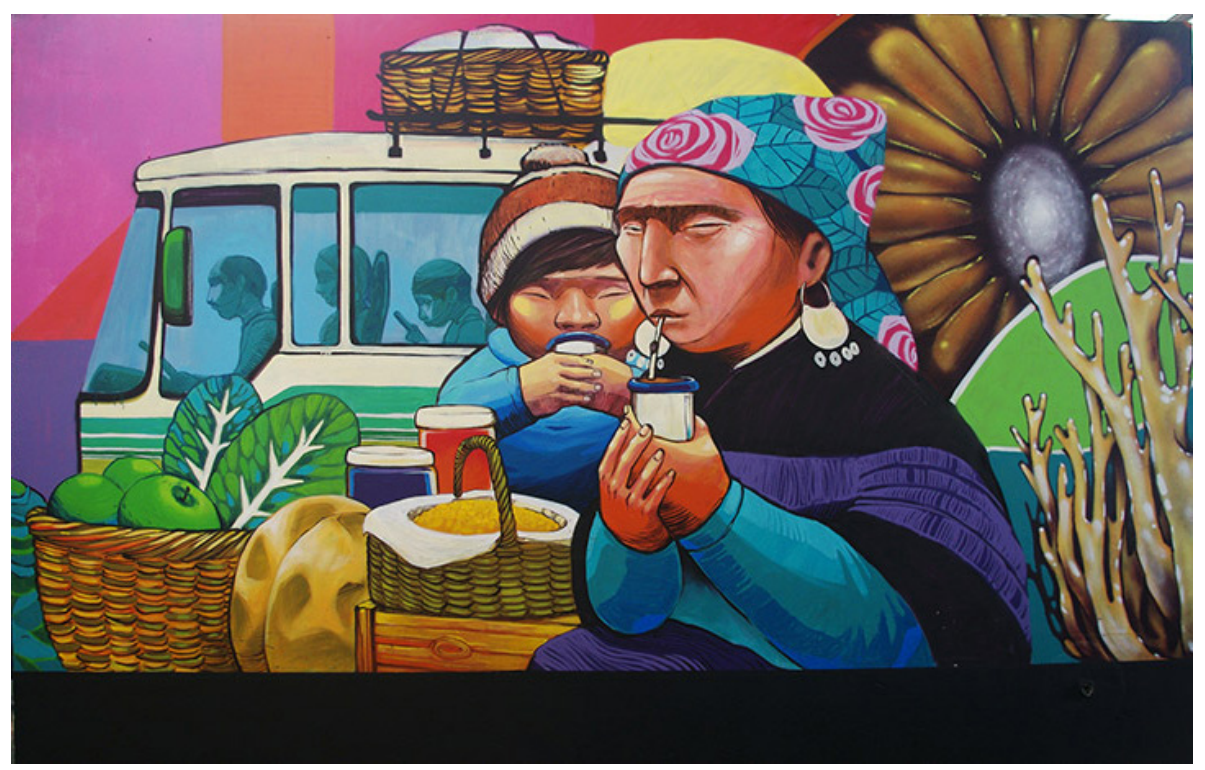

Figura 3. Mural de la Feria Pinto, Temuco. Fuente: Alapinta, Proyecto Feria Pinto-Temuco (tomada de www.alapita.cl).

Mural of the Feria Pinto, Temuco Source: Alapinta, Feria Pinto-Temuco (taken from www.alapinta.cl). 


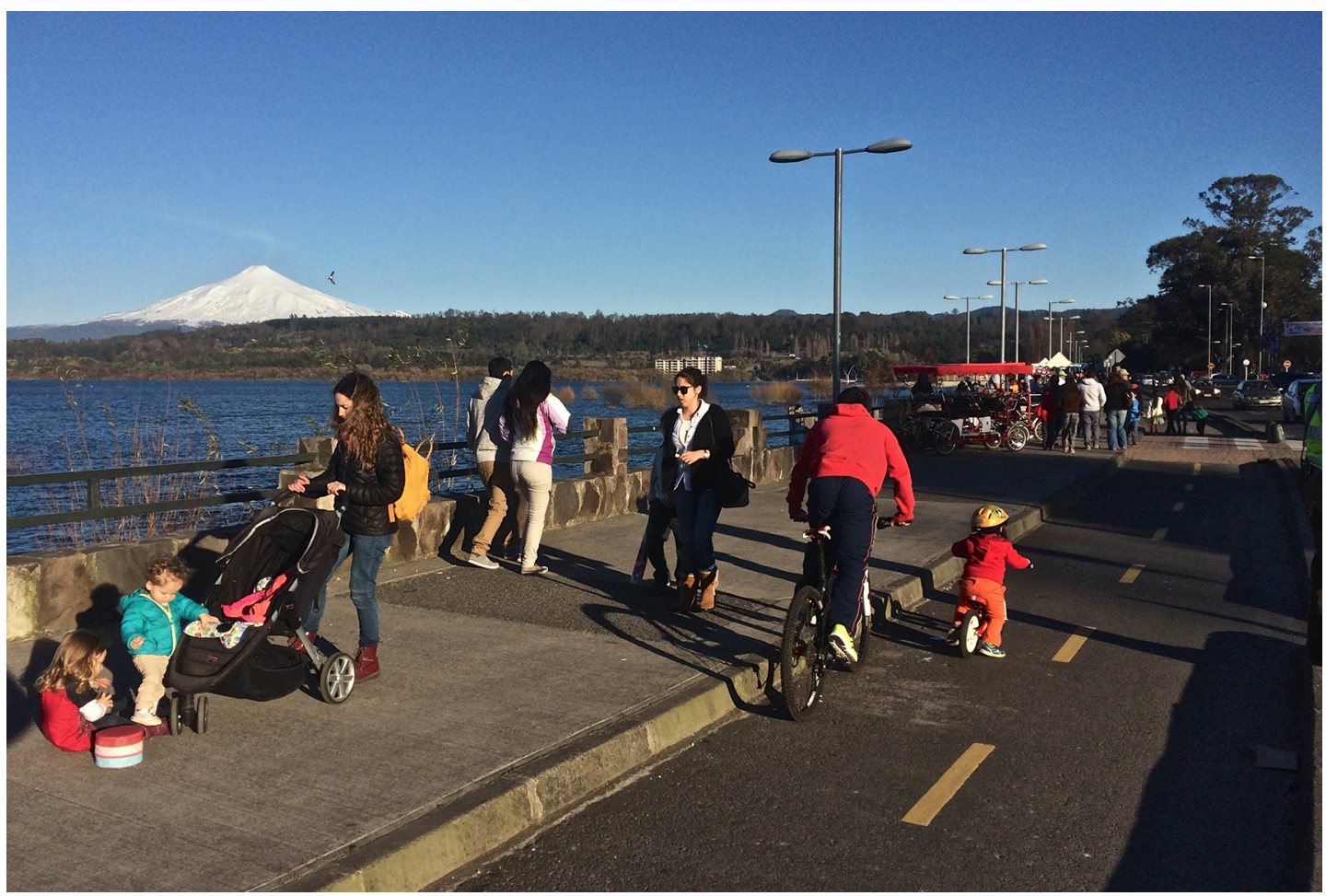

Figura 4. Costanera de Villarrica. Fuente: Elaboración propia. Lakefront of Villarrica. Source: Own Elaboration.

Principalmente ellos resaltan una falta de valoración del entorno natural próximo a la ciudad, donde domina una construcción sociocultural marcada por la desconexión (Salazar y Jalabert 2016). Esto, desde una perspectiva representacional genera la idea de que el paisaje no es valorado, tal como nos señala un entrevistado: "No lo valoran, no lo ven" (hombre, profesor, Villarrica). Del tal modo, desde esta perspectiva lo que caracteriza a la ciudad respecto a su entorno es un desinterés por el paisaje natural: "llama la atención el desinterés, el abandono, la poca consideración" (hombre, profesor, Villarrica).

Sin embargo, desde discursos de dirigentes de organizaciones mapuches de la comuna de Villarrica, emerge como contraste la necesidad de visibilizar las perspectivas de respeto y cuidado por las entidades presentes en el paisaje, destacando el Ruka pillán (volcán Villarrica) y el Mallowelafquén (lago Villarrica).

La cordillera, y las zonas altas, por ejemplo, son entendidas y vividas mediante el movimiento: el arriado de ganado desde las zonas bajas (tradicionalmente llamadas veranadas) y la recolección de piñones (fruto de la araucaria o pehuén) son una práctica común. No obstante, más que entender estas entidades y prácticas como fenómenos separados de lo urbano, son más bien parte de una vida en -y movimientos por- un territorio extendido y relacional. La ciudad se encuentra emplazada en el paso cordillerano hacia la pampa argentina, siendo parte del desplazamiento de población mapuche.

Siguiendo la relevancia de los desplazamientos, actualmente la movilidad de la población rural también se da por su necesidad de comercializar sus productos -producidos o recolectados- en Villarrica; por la necesidad de abastecerse de diversos recursos; o bien para ir a las oficinas de servicios institucionales presentes en la ciudad, principalmente las oficinas de CONAF (Corporación Nacional Forestal), INDAP (Instituto de Desarrollo Agropecuario) y el SAG (Servicio Agrícola Ganadero), lo cual es señalado por dirigentes entrevistados por diferentes lugares de la ciudad. De tal modo, es necesario reconocer desde una perspectiva dinámica del paisaje, la situación espacial de encuentro en el espacio urbano de población rural que viene del campo con una 
participación activa en la ciudad. En este sentido, la perspectiva mencionada respecto a la falta de una valoración ambiental de la población -como juicio general articulado desde un discurso representacional del paisaje- opaca las perspectivas relacionales de paisaje que han constituido históricamente el paso de "la Villa Rica" (Curilaf 2015), como una zona donde el Ruka pillán y el Mallowelafquén han acompañado el sentido de lugar desde las prácticas en movimiento.

\section{Conclusiones}

En este artículo se ha analizado una aproximación al paisaje en las principales ciudades de La Araucanía por medio del sentido de lugar. La principal reflexión analítica que nos entrega el ejercicio es el paso de un entendimiento del paisaje como representación estática a un proceso relacional y territorialmente anclado. El paisaje en ciudades de La Araucanía, antes que estar vinculado únicamente a elementos visuales y/o estéticos del medio ambiente circundante, es construido dinámicamente por medio de relaciones territoriales que se expresan en el espacio urbano y los sentidos de lugar. Esto permite observar con una mayor amplitud y profundidad no solo las dinámicas socioespaciales de las ciudades que son constitutivas de paisajes en movimiento, sino también identificar y territorializar diversas formas de comprender el paisaje. En términos epistemológicos, la aproximación del artículo resalta la relevancia de territorializar la interculturalidad y, en este caso, las formas de abordar el estudio del paisaje.

A raíz de la discusión y los hallazgos emergen dos implicancias importantes para el estudio del paisaje y la interculturalidad de aquellas ciudades fuertemente asociadas a territorios con alta población indígena, tal como sucede en los sistemas urbanos de La Araucanía. Primero, la interculturalidad urbana, más que un fenómeno en sí mismo, está estrechamente vinculada a prácticas y significados territorialmente complejos, a flujos de vida que no distinguen necesariamente entre categorías "urbano" o "rural". Esto no solo nos lleva a trascender dicotomías urbano-rural definidas a priori teóricamente o maximizadas por fines políticos o económicos -como es el caso de Angol- sino también a valorar la importancia que tiene comprender el territorio desde una perspectiva relacional. En este sentido, en términos de planificación y generación de políticas públicas territoriales, la ciudad debe ser entendida como parte de las dinámicas territoriales y las prácticas interculturales en el espacio.

Segundo, un entendimiento del paisaje desde una perspectiva representacional presenta el riesgo de invisibilizar las prácticas espaciales que -como manifestación del mismo proceso de habitar- son parte de la configuración dinámica del paisaje. En los casos estudiados se da cuenta de ciertas representaciones del paisaje que se tienden a jerarquizar, trazando y cultivando criterios que opacan la diversidad constitutiva del territorio de estudio. En las ciudades analizadas los diagnósticos sobre la falta de valoración ambiental del paisaje plantean el riesgo de posicionarse desde una perspectiva representacional y jerárquica. Como consecuencia, se invisibilizan prácticas como la recolección de lahuen en el cerro Nielol de Temuco o la tensión intercultural en el entorno de Angol. Esto se debe al predominio de una perspectiva visual del paisaje que genera discursos y valoraciones desancladas de la experiencia de vida en el paisaje.

Por el contrario, los sentidos de lugar ponen en entredicho estas representaciones del paisaje. $\mathrm{La}$ experiencia frente al agua en Angol da cuenta de cómo ha sido utilizado el espacio desde una perspectiva relacional. Los sentidos que suscita la falta de agua ponen en conexión la ciudad con el territorio otorgando una clave espacial respecto al carácter emplazado del conflicto. Los desplazamientos por la cordillera en Villarrica dan cuenta de las dinámicas territoriales extendidas. Justamente, emerge como hallazgo relevante para los sentidos de lugar y prácticas interculturales, la crucialidad que tiene el movimiento para una perspectiva relacional de paisaje. Las prácticas de recolección operan en un territorio extendido, con una participación activa en el espacio urbano. En este sentido, las prácticas en el territorio dan vida a perspectivas relacionales del paisaje. De este modo, se vuelve fundamental profundizar respecto a la experiencia espacial en las prácticas de vida y las implicancias que esto tiene en la formación relacional del paisaje.

Agradecimientos: Esta investigación ha sido financiada por los siguientes fondos públicos: FONDECYT de Iniciación No 11130519 , Proyecto CONICYT/FONDAP N ${ }^{\circ} 15110020$ (CEDEUS), Proyecto CONICYT/FONDAP No 15110006 (CIIR). Se agradece a los pares evaluadores de esta revista por sus valiosas contribuciones. 


\section{Referencias Citadas}

Abarca, G. 2002. Mapuches de Santiago. Rupturas y continuidades en la recreación de la cultura. Revista de La Academia 7:105-120.

Aliste, E. y A. Núñez 2015. Las fronteras del discurso geográfico: el tiempo y el espacio en la investigación social. Chungara Revista de Antropología Chilena 47:287-301.

Altman, I. y S.M. Low (eds.) 1992. Place Attachment. Plenum Press, New York.

Anderson, J. 2004. Talking whilst walking: a geographical archaeology of knowledge. Area 36:254-261.

Antileo, E. 2007. Mapuche y Santiaguino. El Movimiento Mapuche en torno al Dilema de la Urbanidad. Nuke MapuFórlaget, Santiago.

Antileo, E. 2010. Urbano e Indígena Diálogo y Reflexión en Santiago Warria (Working Paper Series 31), Ñuke Mapuförlage.

Antileo, E. 2013. Políticas indígenas, multiculturalismo y enfoque estatal indígena urbano. Revista de Historia Social y de las Mentalidades 17:133-159.

Aravena, A., N. Gissi y G. Toledo 2005. Los mapuches más allá y más acá de la frontera: identidad étnica en las ciudades de Concepción y Temuco. Sociedad Hoy 8:117-132.

Aravena, A. 2005. Identidades indígenas urbanas en el tercer milenio: identidades étnicas, identidades políticas de los Mapuche-warriache de Santiago de Chile. Ponencia presentada en el I Congreso Latinoamericano de Antropología, Rosario.

Bello, Á. 2002. Migración, identidad y comunidad mapuche en Chile: entre utopistas y realidades. Asuntos Indígenas 3-4:40-47.

Bello, Á. 2011. Espacio y Territorio en Perspectiva Antropológica. El Caso de los Purhépechas de Nurío y Michoacán en México. Universidad Católica de Temuco, Temuco.

Booth, R. 2010. "El paisaje aquí tiene un encanto fresco y poético". Las bellezas del sur de Chile y la construcción de la nación turística. HIb. Revista de Historia Iberoamericana 3:10-32.

Berque, A. 2009. El Pensamiento Paisajero. Biblioteca Nueva, Madrid.

Bustos, B., M. Prieto y J. Barton 2015. Ecología Política en Chile. Naturaleza, Propiedad, Conocimiento y Poder. Editorial Universitaria S. A., Santiago.

Camus, P. y M.E. Solari 2008. La invención de la selva austral: bosques y tierras despejadas en la cuenca del río Valdivia (siglos XVI-XIX). Revista de Geografía Norte Grande 40:5-22.

Corsín Jiménez, A. 2003. On space as a capacity. Journal of the Royal Anthropological Institute 9:137-153.

Cosgrove, D.E. 1984. Social Formation and Symbolic Landscape. Croom Helm, London.

Cresswell, T. 2004. Place: An Introduction. Wiley-Blackwell, Oxford.

Curilaf, L. 2015. Kurarewe en el Boquete del Trankura. Condor Blanco Ediciones, Santiago.

Di Giminiani, P. y M. Fonck 2015. El paisaje como proceso de vida: experiencias de domesticación del bosque en el sur de Chile. Revista de Geografía Norte Grande 61:7-24.
Escobar, A. 2001. Culture Sits in Places: Reflections on Globalism and Subaltern Strategies of Localization. Political Geography 20:139-174.

Escobar, A. 2008. Territories of Difference: Place, Movements, Life, Redes. Duke University Press, Durkham.

Evans, J. y P. Jones 2011. The walking interview: Methodology, mobility and place. Applied Geography 31:849-858.

Feld, S. y K.H. Basso (eds.) 1996. Senses of Place. School for Advanced Research Press, Santafe.

Flores, J. 2012. La Araucanía y la construcción del sur de Chile, 1880-1950. Turismo y vías de transporte. Scripta Nova. Revista Electrónica de Geografía y Ciencias Sociales 418 (12).

Geertz, C. 2000. La Interpretación de las Culturas. Gedisa, Barcelona.

Gupta, A. y J. Ferguson 1992. Beyond "Culture": Space, identity, and the politics of difference. Cultural Anthropology 7:6-23.

Hirsch, E. 1995. Landscape: Between place and space. En The Anthropology of Landscape: Perspectives on Place and Space, editado por E. Hirsch y M. O'hanlon, pp. 1-30. University Press, Oxford.

Ingold, T. 2000. The Perception of the Environment. Routledge, London.

Ingold, T. y J. Lee 2007 [2006]. Fieldwork on foot: Perceiving, Routing, Socializing. En Locating the Field: Space, Place and Context in Anthropology, editado por P. Collins y S. Coleman, pp. 67-86. Berg, New York.

Jackson, J.B. 1984. Discovering the Vernacular Landscape. Yale University Press, New Haven.

Leff, E. 2010. Imaginarios Sociales y Sustentabilidad. Cultura y Representaciones Sociales 5:42-121.

Maldonado, C. y C. Del Valle 2016. Episteme decolonial en dos obras del pensamiento mapuche: Re-escribiendo la interculturalidad. Chungara Revista de Antropología Chilena 48:319-330.

Manzo, L.C. y D.D. Perkins 2006. Finding common ground: the importance of place attachment to community participation and planning. Journal of Planning Literature 20:335-350.

Martínez, N. 2012. Tierra, territorio y territorialidad mapuche: producción de espacio y formación de subjetividades. Revista Geográfica del Sur 3:15-36.

Massey, D. 2005. For Space. SAGE, London.

Mignolo, W. 2010. Desobediencia Epistémica: Retórica de la Modernidad, Lógica de la Colonialidad y Gramática de la Descolonialidad. Del Signo, Buenos Aires.

MINISTERIO DE DESARROLLO SOCIAL 2013. División Social, Departamento de Información Social. Encuesta CASEN.

Molina, R. 1995. Reconstrucción de los etno-territorios. En Tierra, Territorio y Derecho Indígena, editado por J. Aylwin, H. Carrasco y Ch. Martínez, pp. 111-118. IEI-UFRO, Temuco.

Nogué, J. 2008. El Paisaje en la Cultura Contemporánea. Biblioteca Nueva, Madrid. 
Nogué, J. 2014. Sentido del lugar, paisaje y conflicto. Geopolítica(s) 5:155-163.

Panofsky, E. 1991. Perspective as Symbolic Form. Zone Books, New York.

Pinto, J. 2003. La Formación del Estado y la Acción y el Pueblo Mapuche. De la Inclusión a la Exclusión. Dibam, Santiago.

Proshansky, H.M., A.K. Fabian y R. Kaminoff 1983. Placeidentity: Physical world socialization of the self. Journal of Environmental Psychology 3:57-83.

Relph, E. 1976. Place and Placelessness. Pion, London.

Salas Astrain, R. 2014. Debates teórico-metodológicos acerca de reconocimiento e interculturalidad. Revista Faro 2:55-65.

Salazar, G. y D. Jalabert 2016. Towards a landscape ecosophy. Interpreting how the inhabitants of the Villarrica-Pucón urban system in the Araucanía Region of Chile perceive and relate with the dynamics of landscape. Urbe. Revista Brasileira de Gestão Urbana 8:28-41.

Sauer, C. 1925. The morphology of landscape. University of California Publications in Geography 2:19-54.

De Souza Santos, B. de S. 2010. Descolonizar el Saber, Reinventar el Poder. Ediciones Trilce, Montevideo.
Skewes, J.C., D. Guerra y C. Henríquez 2014. Patrimonio y paisaje: dos formas de ensamblar naturaleza y cultura en la cuenca del río Valdivia, sur de Chile. Chungara Revista de Antropología Chilena 46:651-668.

Soja, E.W. 1996. Thirdspace: Journeys to Los Angeles and Other Real-and-Imagined Places. Blackwell, Oxford.

Thiers, J. 2014. Santiago mapuche. La dimensión indígena del espacio urbano en Chile. Scripta Nova 493(47).

Thrift, N. 2008. Non-Representational Theory: Space, Politics, Affect. Routledge, London.

Tilley, C.Y. 1994. A phenomenology of Landscape: Places, Paths, and Monuments. Berg, Oxford.

Tuan, Y. 1974. Topophilia: A Study of Environmental Perception, Attitudes, and Values. Prentice-Hall, Englewood Cliffs, New Jersey.

Vergara, J. y R. Foerster 2002. Permanencia y Transformación del Conflicto Estado-mapuches en Chile. Revista Austral de Ciencias Sociales 6:35-46.

Walsh, C. 2012. Interculturalidad Crítica y (de)Colonialidad. Ensayos desde Abya Yala. Abya Yala, Quito.

\section{Notas}

1 En la actualidad esta perspectiva ha persistido en mayor o menor grado y se ha complementado con un imaginario mediático asociado a conflictividad (Vergara y Foerster 2002).

2 Las referencias al paisaje son constante en la región de La Araucanía, siendo común ver en las calles expresiones artísticas de especies nativas, como las araucarias en la cordillera, o la historia humana relacionada al paisaje tal como lo expresan los murales efectuados por la agrupación Alapinta de Villarrica, ilustrados en el presente trabajo. El presente escrito es deudor del conocimiento cotidiano que tales obras enseñan desde las paredes. 